\title{
Design and development of a kind of hook opened under the loading
}

\author{
LIU Qing-kui ${ }^{1,}$, CHEN Yong-hua ${ }^{2, b}$ \\ ${ }^{1}$ College of Automation \& Electronic Engineering, Qingdao University of Science \& Technology, \\ Qingdao 266042, China \\ ${ }^{2}$ Institute of Oceanology, Chinese Academy of Sciences, Qingdao 266071, China \\ aliuqk5102@163.com, ${ }^{b}$ chenyonghua@qdio.ac.cn
}

Keywords: hook; safety pin; pull rope.

Abstract. This paper introduces a kind of hook which can be opened under the loading. The mechanical structure and working principle of the hook is elaborated. In addition to the advantages of the hook which can be opened under the loading condition to release the weight, the hook can be controlled in the remote distance using the pull rope. Besides, because the safety pin has been inserted in the hook, the safety pin will be pulled out to open bar only when the hook can not be touched, which can prevent false opening.

\section{Introduction}

The marine environment observation often needs the ocean research vessel carrying survey equipment and investigators to arrive at the certain area to get observation of the sea [1]. When the research vessel arrives at the destination, some measuring equipment will be released into water by pulling the deck winch. Lifting a heavy equipment and transfering from one place to another, we often need to release weight without the assist directly by human hands [2-4]. In additon, we often neet to release weight before it can touch the sea surface, such as a lifting winch on ship lifts a certain weight and throws it into the sea, the wire rope on the lifting winch will be pulled free from the weight, as shown in Fig1[5]. Under this circumstance, human hand can not contact with the hook directly, and it is difficult to ensure the weight is thrown into the water with accurate position[6,7]. This paper describes the design of a kind of hook which is opened under the loading by remote control. The hook can hook in open unloading heavy weight, which will be released into the water.

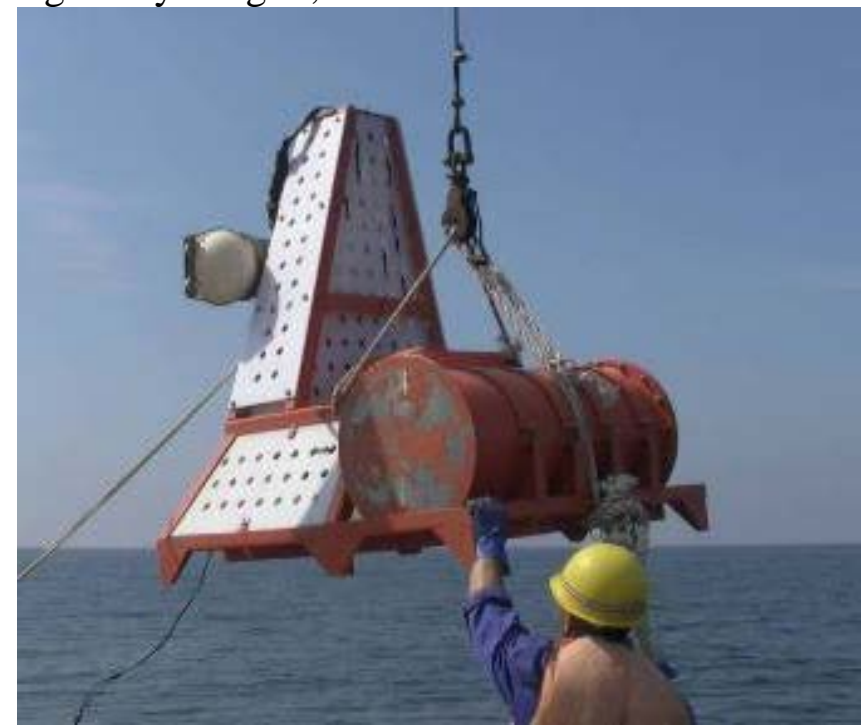

Fig.1 Ordinary hook

\section{Mechanical structure and Working principle}

Hook structure diagram as shown in figure 2 


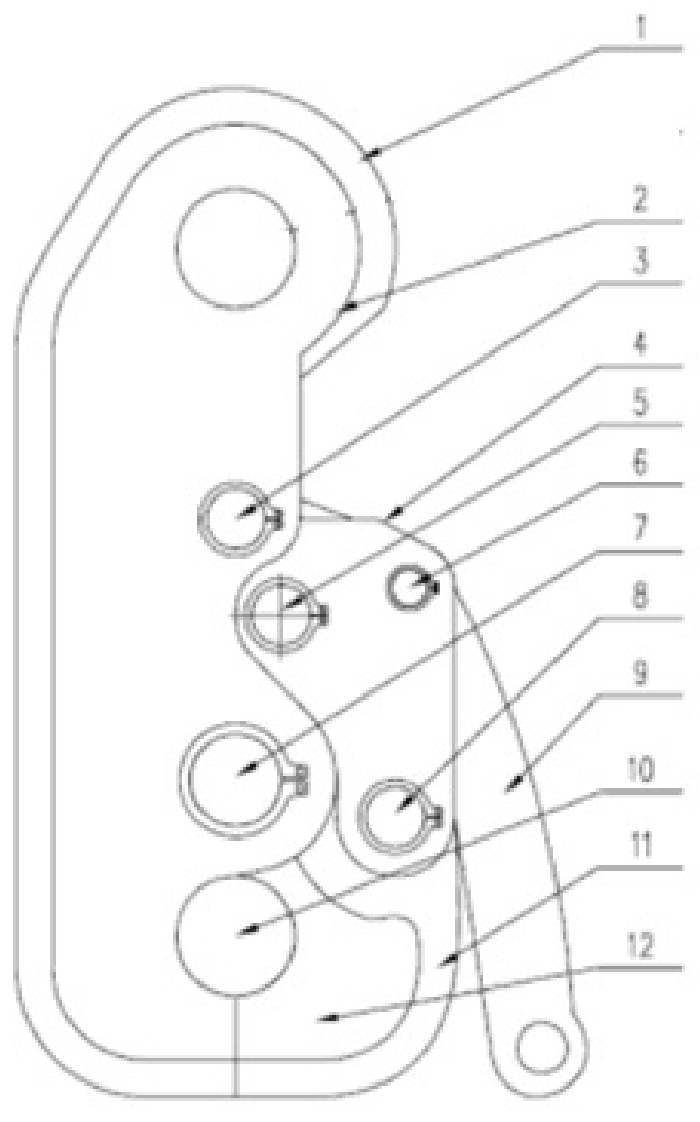

Fig.2 Hook structure diagram

1.Hook framework;2.Main lining board;3.spindle II;4.Pull lining board;5.spindle III;6.Safety pin;7.Spindle I;8.Spindle IV;9.Open bar;10.Hanging hole;11.On-off hook;12. Reinforcement lining board.

The device is a kind of hook opened under the loading, including hook framework1, on-off hook11 and Open bar9, main lining board 2 is installed symmetrically on the front and back side of hook framework1, on-off hook is installed under the side of hook framework 1 and links with two main lining boards by the Spindle I 7. Reinforcement lining board is installed symmetrically on the front and back side of on-off hook 11 just like hook framework1.Hanging hole 11 that can be opened is made up with on-off hook I7 and hook framework, it is arranged with a pair of pull lining boards 4 by side as the open bar is arranged between the two pull lining boards. The open bar and main lining board 2 are linked with the spindle II which is connected with the spindle III 5 on the other side, the spindle III 5 passes through two pull lining boards 4 and open bar 9,a on-off hook 11 extends into the outer side of the upper end of the two pull lining board 4 and connected by a spindle IV 8 . When the side of on-off hook 11 is connected with the side of hook framework, a safety pin can be inserted between the open bar and two main lining boards 2.Both ends of the spindle I 7, II 3, III 5 and IV 8 are respectively provided with grooves. On the upper of the open hook unloading heavy weight is arranged with the lifting fixing hole 13 . The hanging hole 10 is composed of the hook framework and on-off hook 11 as the left and right half hanging holes are drilled on the side of them. The free end of the open bar 9 is opened with a pull rope perforation and is provided with a pull rope.

When used, the operation steps of the hook are as follows:

(1)When we need lift weights, it will be necessary for turning the open bar 9 to the lower end of the hook, making the hanging hole 10 to be closed and inserting the safety pin 6 .

(2)Then the shackle should be put into the hanging hole 10 and hook the weight.

(3) When the weight is hoisted up to certain height, we should pull out the safety pin 6.

(4) Keep the weight to the position, pull the rope to open the hanging hole 10, release the weight and complete the process of hanging.

Because of the limiting function of the safety pin 6 , the hook will not be opened to release the 
weight suddenly and unconsciously when under the loading condition; safety is ensured through elaborate design. But when the hook needs to be opened at high altitude, it is difficult for the operator to pull out the safety pin 6 . In order to solve this above problems, we have designed an auxiliary structure. A strong electromagnet, arranged on the side of one side of the pull lining board and the safety pin 6 , is vertically connected by strong magnet wire and the external power supply. The safety pin 6 toward the end of a strong magnet rope and one end of the rope is connected with a pull at the edge of the pull lining board 4.when the hook ,keeping closed, is moved to the position that need to release the weight, the utility model only needs to make a strong electromagnetic switch power through an electric wire and a strong magnetic force is generated at one end of the safety pin 6 as a large pulling force is generated at the end of the safety pin 6 to pull it from the pull lining board 4 and the open bar. Then the electromagnet can be cut off and the safety pin 6 falls into a free state. With the function of the rope, the safety pin 6 is freely hung on the pull lining board 4 and the unlocking is realized. Thus, the open bar 9 can be rotated freely.

The working principle of the hook is to make the pull lining board and the on-off hook linkage by pulling the open bar and then the hanging hole is opened, so the hook can be achieved to release. On the front and rear side of the on-off hook are symmetrically installed reinforcement lining board, which can improve the bearing ability of the on-off hook and the overall strength of the hook. Both ends of the spindle I, II, III and IV are respectively provided with grooves that can prevent the occurrence of the four rotating shaft separation in the lifting process and ensure the safety of hoisting. On the upper of the open hook unloading heavy weight is arranged with the lifting fixing hole which can make it easy for the rope of the crane connect with the hook. The hanging hole 10 is composed of the hook framework and on-off hook 11 as the left and right half hanging holes are drilled on the side of them, this design is reasonable, so that the opening and closing of the hanging hole is simpler and faster. The free end of the open bar 9 is opened with a pull rope perforation and is provided with a pull rope, the utility model is convenient to pull the open bar. A strong electromagnet, arranged on the side of one side of the pull lining board and the safety pin 6 , is vertically connected by strong magnet wire and the external power supply.

Design of the hook closed and open state as shown in Fig.3 and Fig.4.

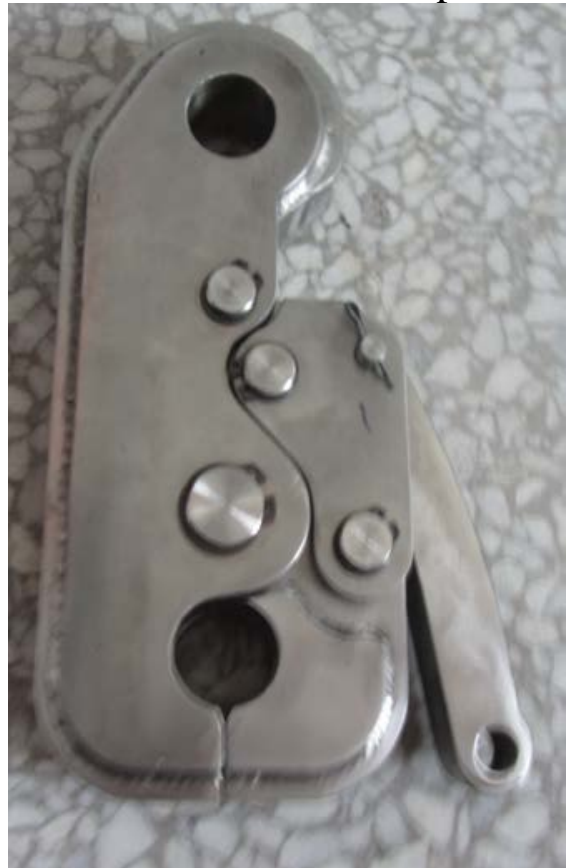

Fig.3 Hook closed

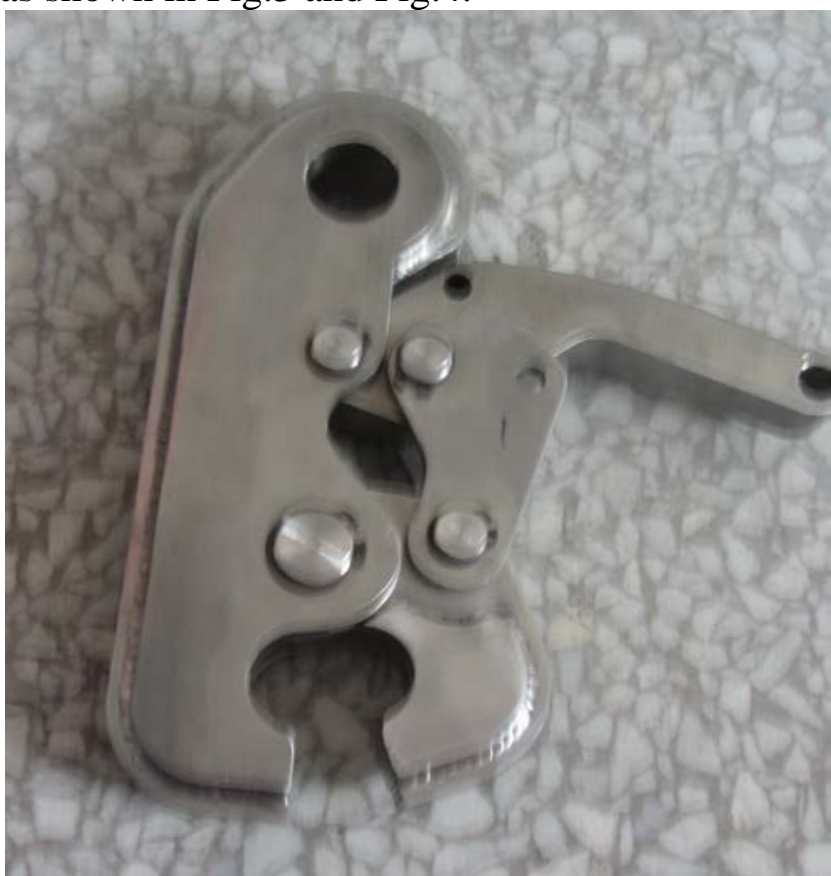

Fig.4 Hook open

\section{Summary}

The hook opened under the loading has the following advantages:

(1) The hook can be opened in the unloading condition, which using the leverage principle of the 
open bar and the motion transmissibility of the pull lining board and the weight can be released.

(2) Remote control can be achieved and with the help of the pull rope ,the rotation of the pull rod can be controlled at a distance.

(3) The relative safety, the safety pin has been inserted in the hook in the process of lifting heavy objects and the safety pin is pulled out only when the hand is about to reach it, only at this time you can pull the open bar and it is effective to prevent the wrong open.

\section{Acknowledgements}

The project was financially supported by Key research and development of science and technology Program (Grant No. 2016YFC1402602), by major scientific research equipment R \& D projects of Chinese Academy of Sciences (Grant No. YZ201522) and by strategic leading science and technology special project of Chinese Academy of Sciences(Class A)(Grants No. XDA11040306.

\section{References}

[1]WANG Xin, WANG Feng-quan, WANG Xi-liang. Simulation of a new hydraulic decoupling system based on ADAMS [J]. Construction Machinery.2006,(05):59-61.

[2]JIA Li-shuang, ZHANG Xuan-ming, FENG Zhi-tao etc. The design of deep sea acoustic response releaser [J].Ocean Technology.2013. (02):90-93

[3] Hall S J. Closed areas for fisheries management-the case consolidates.[J]. Trends in ecology \& evolution, 1998, 13(8).

[4] Hilborn Ray. Moving to sustainability by learning from successful fisheries.[J]. Ambio, 2007, 36(4).

[5] Hughes Steven E, Woodley Christopher. Transition from open access to quota based fishery management regimes in Alaska increased the safety of operations.[J]. International maritime health, 2008, 58(1-4).

[6] Sale Peter F, Cowen Robert K, Danilowicz Bret S et al.. Critical science gaps impede use of no-take fishery reserves.[J]. Trends in Ecology \& Evolution, 2006, 20(2).

[7] Fogarty. Essential habitat, marine reserves and fishery management. [J]. Trends in ecology \& evolution (Personal edition), 1999, 14(4). 